(1)

CrossMark

\title{
In addition to mTOR and JAK/STAT, NLRP3 inflammasome is another key pathway activated in sarcoidosis
}

\author{
Nicolas Riteau (1) ${ }^{1}$ and Jean-François Bernaudin ${ }^{2,3,4}$ \\ Affiliations: ${ }^{1}$ CNRS, INEM-UMR7355, University of Orleans, Orleans, France. ${ }^{2}$ Sorbonne Université, Paris, \\ France. ${ }^{3}$ INSERM UMR 1272 Université Paris 13, Bobigny, France. ${ }^{4}$ Pneumology Dept, Hôpital Avicenne APHP, \\ Bobigny, France.
}

Correspondence: Nicolas Riteau, Immunologie et embryologie moleculaires CNRS - INEM - UMR 7355 Institut de Transgénose 3B, rue de la Férollerie, 45071 Paris, France. E-mail: nicolas.riteaudacnrs-orleans.fr

@ERSpublications

The study of Huppertz and co-workers addresses the role of the NLRP3 inflammasome in sarcoidosis. Employing both mouse model and human samples, they provide evidence of NLPR3 inflammasome activation and increased IL-1ß production in lung granulomas. http://bit.ly/32a $4 \mathrm{GsI}$

Cite this article as: Riteau N, Bernaudin J-F. In addition to mTOR and JAK/STAT, NLRP3 inflammasome is another key pathway activated in sarcoidosis. Eur Respir J 2020; 55: 2000149 [https://doi.org/10.1183/ 13993003.00149-2020].

Sarcoidosis is a rare "old disease" first described over a century ago, known as a multifaceted systemic multiorgan inflammatory granulomatosis characterised by the presence of non-necrotising epithelioid granulomas [1, 2]. In many ways sarcoidosis remains a mysterious disease with numerous unsolved knowledge gaps. The still unknown aetiology/aetiologies of sarcoidosis is one of these gaps, i.e. the disease develops in genetically predisposed individuals following as-yet-unknown antigen exposure. Various triggers or causes have been suspected, including bacterial agents (such as Propionibacterium acnes or mycobacterial antigens) or environmental particulates (such as crystalline silica), but their true identity and mechanisms of action are still not elucidated [1-3]. The second main gap regards the nature of the granuloma foundation, the hallmark of the disease, as its mechanisms related to complex immunopathogenesis are only partially understood [1-3]. Furthermore, even though the highly variable course of the disease is well documented, disease evolution is still quite unpredictable for a given patient. In many cases, tissue granulomas resolve spontaneously, while in others they persist or reoccur and require treatment; usually prednisone alone, or other drugs such as immunosuppressants $[1,2,4]$. The current therapeutic monitoring is hence mainly based on empirical knowledge and not on well-identified pathogenesis mechanisms. Therefore, a better knowledge of the intracellular pathways potentially activated is needed.

As emphasised by R.P. Baughman and D. Valeyre in the preface of the sarcoidosis book they have coordinated, "The world of sarcoidosis is changing on many fronts" [5]. The disease phenotypic variability has been recently challenged at both individual and epidemiological levels. Data obtained from the GenPhenReSa (Genotype-phenotype relationship in sarcoidosis) European project allowed a smart subgrouping of sarcoidosis patients according to main organ clusters [4]. According to their clinical patterns, 2163 European patients were distributed in five distinct subgroups according to predominant organ involvement. In addition, three geo-epidemiological factors, i.e. geolocation, ethnicity and personal environment, from 128955 patients were compared on a basis of published studies reporting at least 
100 patients each and showed different patterns among people of White, Black and Asian origin [6]. The authors concluded that "Sarcoidosis is probably one of the systemic diseases with the greatest influence of geo-epidemiological factors on the frequency and phenotypic expression." These results emphasise that sarcoidosis is not a univocal disease. Its variability may reflect genetic background and environmental exposure differences as well as the socioeconomic status of patients. Therefore, one can postulate that sarcoidosis pathogenesis implies the activation of different intracellular pathways.

The recent identification of significant key cellular pathways can be considered as the beginning of an answer related to the complexity of sarcoidosis. Indeed, the mammalian target of rapamycin (mTOR) pathway [7], the Janus kinase/signal transducers and activators of transcription (JAK/STAT) pathway $[8,9]$ and the NLRP3 inflammasome pathway (described in this issue of the European Respiratory Journal [10]) have been brought to light within the past 3 years. LinKE et al. [7] showed that activation of the metabolic checkpoint kinase mTORC1 in macrophages by $T s c 2$ deletion resulted in a model of sarcoid-like granulomatosis in mice. In addition, the authors identified activated mTORC1 signalling in one third of granulomas obtained from 27 biopsies from patients. These results were confirmed by CALENDER et al. [11] in genetic studies on five French families, which led to the identification of genes enriched in the regulation of mTOR signalling pathway and autophagy. As a consequence, a clinical trial targeting mTOR in a pilot study of sirolimus inhibitors in sarcoidosis with cutaneous affection is ongoing (EudraCT Number: 2017-004930-27). A second breakthrough came from DAMSKY et al. [8], who successfully treated a refractory cutaneous sarcoidosis patient with JAK2 oral inhibitors. The authors also assessed the role of activated JAK-STAT signalling in these granulomatous lesions from consecutive biopsies of this patient and 21 archived tissue samples. Concomitantly, Rotenberg et al. [9] reported a dramatic improvement in a patient with long-standing sarcoidosis with multivisceral flares refractory to corticosteroids associated with JAK2-mutated polycythaemia using the ATP-competitive JAK inhibitor ruxolitinib. Since, JAK inhibitors are considered promising for a new era of targeted treatment for granulomatous disorders [12], and clinical trials are ongoing mostly on refractory cutaneous sarcoidosis (ClinicalTrials.gov Identifier: NCT03910543).

In this vein, HUPPERTZ et al. [10] report on the role of NLRP3 inflammasome in sarcoidosis adds a new stone. A role for interleukin (IL)-1 $\beta$ secretion by macrophages in sarcoidosis has been suggested by multiple studies almost 30 years ago but quite overlooked since [13-15]. However, the IL-1 $\beta$ family has recently regained interest in part due to the molecular delineation of the inflammasome pathway. Since its discovery in 2002, the inflammasome is regarded as a key inflammatory pathway with major implications in various conditions and as a potential target for treatments $[16,17]$. The inflammasome was first coined in 2002 by the group of Jürg Tschopp [18]. Employing 293T cell line or cell extracts from human monocytes-derived cell line (THP1), MARTINON et al. [19] discovered that IL-1 $\beta$ maturation requires caspase-1 (Casp-1) activity through NLRP1-based caspase-activating complex that they call the inflammasome. The NLRP3 inflammasome was discovered by the same group in a study showing that patients suffering from Muckle-Wells syndrome exhibit gain-of-function mutations in NLRP3 gene leading to constitutive macrophage inflammasome activation and mature IL-1 $\beta$ production [20]. Uric acid crystal was the first reported activator of the NLRP3 inflammasome, leading to gout disease.

In their manuscript, HUPPERTz et al. [10] have provided evidence of inflammasome activation and IL-1 $\beta$ expression in sarcoid granulomas by showing consecutive sections of transbronchial biopsy from a sarcoid patient in which CD68 expressing cells (used as a monocyte marker) coexpressed active Casp-1 and IL-1ß. In addition, IL- $1 \beta$ staining score positively correlates with disease severity. They further show that while untreated bronchoalveolar lavage (BAL) cells from healthy volunteers or patients with scleroderma secrete basal levels of IL-1 $\beta$, cells from severe sarcoidosis patients display increased IL-1 $\beta$ production. Augmented IL-1 $\beta$ and cleaved Casp-1 productions in BAL cells from sarcoid patients were further confirmed using inflammasome activating conditions in vitro. The authors also report that mRNA levels of miR-223, a micro RNA known to downregulate NLRP3, were decreased in alveolar macrophages sorted from BAL cells, while NLRP3 mRNA expression levels were indeed increased. The study of noncoding RNA in sarcoidosis is a new field of investigation, with potential critical significance in terms of diagnosis and treatment [21].

The authors then performed experiments employing a mouse model of granuloma to mimic some features of human sarcoidosis by injecting trehalose 6,6'-dimycolate (TDM) mixed in incomplete Freund's adjuvant (IFA). TDM (also known as cord factor) is a glycolipid molecule extracted from the cell wall of Mycobacterium tuberculosis or related species. As compared to untreated conditions, BAL cells from TDM treated mice displayed increased IL-1 $\beta$ release following inflammasome-triggering conditions in vitro. Immunohistochemical staining of consecutive lung sections showed that macrophages in the granulomas (Iba-1-positive areas) express IL-1 $\beta$. The authors investigated the role of NLRP3 as well as its downregulator miR-223 by using deficient animals. NLPR3- and miR-223-deficient mice displayed 
decreased or increased lung granuloma counts, respectively, confirming the pathological role of the inflammasome using this in vivo model. Finally, the authors treated wildtype animals with the NLRP3 inhibitor MCC950 [22] or with anti-IL-1 $\beta$ antibody to assess the effects of pharmacological interventions targeting the inflammasome pathway. Both MCC 950 or anti-IL-1 $\beta$ treatments resulted in reduced numbers of pulmonary granuloma in TDM-treated wildtype mice.

While these data strongly suggest that the inflammasome is active in sarcoidosis, additional studies are required to confirm the exact contribution of IL-1 $\beta$ in the disease, especially at early stages. Interestingly, in this manuscript HuPPERTz et al. [10] showed that scleroderma patients with severe lung function impairment display no constitutive NLRP3 inflammasome activation. In contrast, numerous NLRP3 inflammasome activation triggers are potentially involved in sarcoidosis, from viral or bacterial pathogen-associated molecular patterns to endogenous molecules resulting from cell stress/death. For instance, the acute phase protein serum amyloid A, detected in most sarcoid granulomas [23], potentially locally produced by macrophage-derived cells [24], is known to activate the NLRP3 inflammasome by involving the purinergic receptor P2X7 and cathepsin B [25].

Multiple pathways have been described in the pathogenesis of sarcoidosis, including autophagy/mTOR $[7,11]$ and JAK/STAT $[8,9,12]$ signalling pathways and future investigations are required to provide important leads toward the development of effective therapeutic strategies. Targeting the inflammasome using MCC950 [22] or other molecules is currently under deep investigation in multiple conditions [17], such as cardiac sarcoidosis (MAGiC-ART; ClinicalTrials.gov Identifier: NCT04017936). The intersection of granulomatosis and autoinflammatory diseases is a rare occurrence. The field of autoinflammatory disorders expanded significantly in the last decade as IL-1-related autoinflammatory syndromes heterogeneous group of disorders share responsiveness to IL-1 blockers [26]. The results obtained by HUPPERTZ et al. [10] published in this issue of the European Respiratory Journal reinforces the possibility that sarcoidosis may be considered one of the polygenic autoinflammatory diseases [26].

Conflict of interest: None declared.

\section{References}

Valeyre D, Prasse A, Nunes H, et al. Sarcoidosis. Lancet 2014; 383: 1155-1167.

Grunewald J, Grutters JC, Arkema EV, et al. Sarcoidosis. Nat Rev Dis Primers 2019; 5: 45.

Jonsson E, Jarvholm B, Andersson M. Silica dust and sarcoidosis in Swedish construction workers. Occup Med (Lond) 2019; 69: 482-486.

4 Schupp JC, Freitag-Wolf S, Bargagli E, et al. Phenotypes of organ involvement in sarcoidosis. Eur Respir J 2018; 51: 1700991.

5 Baughman RP, Valeyre D. Sarcoidosis: A Clinician's Guide. Elsevier Health Sciences, 2018.

6 Brito-Zeron P, Kostov B, Superville D, et al. Geoepidemiological big data approach to sarcoidosis: geographical and ethnic determinants. Clin Exp Rheumatol 2019; 37: 1052-1064.

7 Linke M, Pham HT, Katholnig K, et al. Chronic signaling via the metabolic checkpoint kinase mTORC1 induces macrophage granuloma formation and marks sarcoidosis progression. Nat Immunol 2017; 18: 293-302.

8 Damsky W, Thakral D, Emeagwali N, et al. Tofacitinib treatment and molecular analysis of cutaneous sarcoidosis. N Engl J Med 2018; 379: 2540-2546.

9 Rotenberg C, Besnard V, Brillet PY, et al. Dramatic response of refractory sarcoidosis under ruxolitinib in a patient with associated JAK2-mutated polycythemia. Eur Respir J 2018; 52: 1801482.

10 Huppertz C, Jäger B, Wieczorek G, et al. The NLRP3 inflammasome pathway is activated in sarcoidosis and involved in granuloma formation. Eur Respir J 2020; 55: 1900119.

11 Calender A, Lim CX, Weichhart T, et al. Exome sequencing and pathogenicity-network analysis of five French families implicate mTOR signalling and autophagy in familial sarcoidosis. Eur Respir J 2019; 54: 1900430.

12 Rosenbach M. Jak-inhibitors offer promise for a new era of targeted treatment for granulomatous disorders. J Am Acad Dermatol 2020; 82: e91-e92.

13 Wewers MD, Saltini C, Sellers S, et al. Evaluation of alveolar macrophages in normals and individuals with active pulmonary sarcoidosis for the spontaneous expression of the interleukin-1 beta gene. Cell Immunol 1987; 107: 479-488.

14 Pueringer RJ, Schwartz DA, Dayton CS, et al. The relationship between alveolar macrophage TNF, IL-1, and PGE2 release, alveolitis, and disease severity in sarcoidosis. Chest 1993; 103: 832-838.

15 Rolfe MW, Standiford TJ, Kunkel SL, et al. Interleukin-1 receptor antagonist expression in sarcoidosis. Am Rev Respir Dis 1993; 148: 1378-1384.

16 Lamkanfi M, Dixit VM. Inflammasomes and their roles in health and disease. Annu Rev Cell Dev Biol 2012; 28: 137-161.

17 Swanson KV, Deng M, Ting JP. The NLRP3 inflammasome: molecular activation and regulation to therapeutics. Nat Rev Immunol 2019; 19: 477-489.

18 Martinon F, Burns K, Tschopp J. The inflammasome: a molecular platform triggering activation of inflammatory caspases and processing of proIL-beta. Mol Cell 2002; 10: 417-426.

19 Martinon F, Petrilli V, Mayor A, et al. Gout-associated uric acid crystals activate the NALP3 inflammasome. Nature 2006; 440: 237-241.

20 Agostini L, Martinon F, Burns K, et al. NALP3 forms an IL-1beta-processing inflammasome with increased activity in Muckle-Wells autoinflammatory disorder. Immunity 2004; 20: 319-325. 
21 Salamo O, Mortaz E, Mirsaeidi M. Noncoding RNAs: new players in pulmonary medicine and sarcoidosis. Am J Respir Cell Mol Biol 2018; 58: 147-156.

22 Coll RC, Robertson AA, Chae JJ, et al. A small-molecule inhibitor of the NLRP3 inflammasome for the treatment of inflammatory diseases. Nat Med 2015; 21: 248-255.

23 Chen ES, Song Z, Willett MH, et al. Serum amyloid A regulates granulomatous inflammation in sarcoidosis through Toll-like receptor-2. Am J Respir Crit Care Med 2010; 181: 360-373.

24 Jumeau C, Awad F, Assrawi E, et al. Expression of SAA1, SAA2 and SAA4 genes in human primary monocytes and monocyte-derived macrophages. PLoS One 2019; 14: e0217005.

25 Niemi K, Teirila L, Lappalainen J, et al. Serum amyloid A activates the NLRP3 inflammasome via P2X7 receptor and a cathepsin B-sensitive pathway. J Immunol 2011; 186: 6119-6128.

26 Pathak S, McDermott MF, Savic S. Autoinflammatory diseases: update on classification diagnosis and management. J Clin Pathol 2017; 70: 1-8. 